\title{
Non-tuberculous mycobacteria: baseline data from three sites in Papua New Guinea, 2010-2012
}

\author{
Serej Ley, ${ }^{a b c}$ Robyn Carter, ${ }^{d}$ Korai Millan, ${ }^{c}$ Suparat Phuanukoonnon, ${ }^{c}$ Sushil Pandey, ${ }^{d}$ Christopher Coulter, ${ }^{d}$ \\ Peter Sibac and Hans-Peter Beck ${ }^{\mathrm{ab}}$ \\ Correspondence to Hans-Peter Beck (email: Hans-Peter.Beck@unibas.ch).
}

Objective: To determine the proportion of non-tuberculous mycobacteria (NTM) in samples of pulmonary tuberculosis (TB) cases from Papua New Guinea who were diagnosed using acid-fast microscopy.

Methods: As part of a case detection study for TB, conducted in three provincial hospitals in Papua New Guinea, sputum samples of suspected tuberculous cases aged 15 years or older were collected from November 2010 to July 2012. Mycobacterial species isolated from sputum and grown in culture were examined to distinguish between NTM and the Mycobacterium tuberculosis complex (MTBC).

Results: NTM were detected in $4 \%(9 / 225)$ of sputum samples grown in culture. Five $(2.2 \%)$ of them were identified as NTM only and four (1.8\%) were identified as mixed cultures containing both MTBC and NTM. Four different NTM species were identified; $M$. fortuitum, $M$. intracellulare, $M$. terrae and $M$. avium.

Discussion: This is the first report from Papua New Guinea identifying NTM in three different locations. As NTM cannot be distinguished from M. tuberculosis through smear microscopy, the presence of NTM can lead to a false-positive diagnosis of tuberculosis. The prevalence of NTM should be determined and a diagnostic algorithm developed to confirm acid-fast bacilli in a smear as $M$. tuberculosis.

A part from the Mycobacterium tuberculosis complex (MTBC), the genus Mycobacterium includes over 120 species of non-tuberculous mycobacteria (NTM). ${ }^{1}$ NTM can be found in the environment, including water and soil, which is the suspected source of occasional infection of humans. Asymptomatic colonization as well as symptomatic disease can be caused by NTM, ${ }^{2}$ including, among others, chronic pulmonary disease with symptoms similar to tuberculosis (TB) such as chronic cough (with or without sputum production), chest pain and weight loss. ${ }^{3,4}$ Different NTM have been associated with different disease presentations. The $M$. avium complex (including $M$. avium and $M$. intracellulare) is most often associated with pulmonary infection. $M$. fortuitum has been associated with pulmonary infection but more often affects the skin, soft tissue or bones. Immunocompromised cases (e.g. human immunodeficiency virus [HIV] positive cases) are susceptible to NTM infection, particularly disseminated M. avium disease. ${ }^{2}$ However, immunocompetent cases with no predisposing conditions can also be affected. ${ }^{5-8}$
Standard first-line anti-TB treatment drugs are less effective against NTM compared to $M$. tuberculosis $(M t b){ }^{2,9}$ and no single regimen for NTM exists to date. Depending on the NTM species, recommendations for treatment regimens include treatment with antibiotics and sometimes even surgical removal of infected tissue. $^{2,10}$ The $M$. avium complex is treated with combination therapy consisting of Clarithromycin, Rifampicin and Ethambutol and should be continued for one year. ${ }^{11}$ While the regimen includes Rifampicin and Ethambutol, two of the standard first-line anti-TB drugs, the length of the TB regimen is not sufficient to address M. avium complex infections. Additionally, Isoniazid (apart from Rifampicin the most potent first-line antiTB drug) has only a limited effect on $M$. avium, ${ }^{9}$ and relapses are common. ${ }^{2}$

Little data are available on the prevalence of NTM infections in TB high-burden countries, but the incidence can nevertheless be substantial. ${ }^{12}$ High TB-burden countries also tend to be resource-poor

\footnotetext{
Swiss Tropical and Public Health Institute, Basel, Switzerland.

University of Basel, Basel, Switzerland.

Papua New Guinea Institute of Medical Research, Goroka and Madang, Papua New Guinea.

Queensland Mycobacterium Reference Laboratory, Pathology Queensland, Brisbane, Australia.

Submitted: 5 May 2015; Published: 24 November 2015

doi: 10.5365/wpsar.2015.6.2.004
} 
countries, and the diagnosis of pulmonary TB is based on the microscopic detection of acid-fast bacilli (AFB) in sputum samples. Smear microscopy cannot distinguish between NTM and Mtb. Mixed infections as well as false-positive TB diagnosis cannot be ruled out. Many diagnostic assays are not optimized to detect different NTM species; if NTM are present in conjunction with $M t b$, the former might remain undetected or cause failure of drug susceptibility testing (DST). ${ }^{13-15}$ Exposure to NTM has been suggested to impact on the efficacy of the Bacille Calmette-Guérin vaccine ${ }^{16}$ and to exhibit cross-reactivity to the tuberculin skin test (TST), leading to increased difficulties in interpreting TST-positive results and evaluating the protection through the only available vaccine against TB. ${ }^{17,18}$

Very little information is available on NTM in Papua New Guinea. Data from a leprosy trial conducted in Karimui (Eastern Highlands Province) in the $1960 s^{19,20}$ as well as a TST sensitivity study conducted in the Marawaka area of the Eastern Highlands of Papua New Guinea ${ }^{21}$ found no evidence for environmental mycobacteria being present in this area. Therefore it was important to investigate the presence of NTM in sputum samples collected in Papua New Guinea. Here we describe the NTM detected and provide baseline information on these bacteria in Papua New Guinea.

\section{METHODS}

As part of a case detection study for TB, conducted between November 2010 and July 2012 in selected provincial hospitals in Papua New Guinea, sputum samples of suspected TB cases aged 15 years or older were collected for laboratory testing. The sampling procedure has been described previously. ${ }^{22}$

Upon diagnosis of TB through AFB Ziehl-Neelson (ZN) microscopy or chest $\mathrm{X}$-ray, sputum samples were decontaminated following Petroff's method; ${ }^{23}$ inoculated into BD Bactec ${ }^{\circledR}$ Mycobacterial Growth Indicator Tube (MGIT) media (Becton, Dickinson and Co., Franklin Lakes, New Jersey, USA); and subsequently sent to the Queensland Mycobacterium Reference Laboratory in Brisbane, Australia for culture. The samples were incubated in the MGIT until they became culture positive (i.e. growth could be detected). A repeat ZN smear was prepared on all culture-positive isolates to confirm the presence of acid-fast organisms. A rapid immuno- chromatographic identification test (SD BIOLINE/BD TB Ag MPT64 Rapid, Standard Diagnostics, Giheunggu, Republic of Korea) was used to confirm the AFB as MTBC.

When the rapid test was negative or the microscopic morphology did not suggest the AFB were MTBC, further molecular analysis was conducted to identify the isolate as NTM or MTBC. In brief, DNA was extracted using crude boil method at $95{ }^{\circ} \mathrm{C}$ for 30 minutes, followed by sonication for 15 minutes. The extracted DNA was then used as a template for polymerase chain reaction (PCR) amplification either according to the GenoType ${ }^{\circledR}$ Mycobacterium Common Mycobacteria line probe kit (Hain LifeSciences, Nehren, Germany) according to the manufacturer's protocol for the GenoType 16S rRNA (Forward primer 5' AGAGTTGGATCCTGGCTCAG; Reverse primer 5' CCTACGAGCTCTTTACG). The amplified product was purified using 4ul EXOSAP-IT (Affymetrix, San Diego, California, USA) and 1 Oul of primary amplification product $\left(37{ }^{\circ} \mathrm{C} 15\right.$ minutes, $80{ }^{\circ} \mathrm{C} 15$ minutes, $40{ }^{\circ} \mathrm{C}$ soak). A repeat gel was run using Invitrogen Bufferless Gel system (ThermoFisher Scientific, Waltham, Massachusetts, USA). The sequencing reaction was performed using the Big Dye Terminator method on ABI3130 sequencer (Distribio, Dudelange, Luxembourg), and the resulting sequences were analysed by comparing them to the National Center for Biotechnology Information Genbank database. In case cultures were identified as MTBC, DST was subsequently performed by the proportion method, ${ }^{24}$ as described previously. ${ }^{25}$ However, if a culture turned out to be NTM, no DST was performed.

Demographic and clinical symptoms of the cases were also collected for analysis. Statistical analysis was carried out with Stata 12.1 (Stata-Corp, College Station, Texas, USA). Excel was used for basic calculations. Due to a small sample size, no statistical analysis for the NTM population was performed.

Ethical approval for this study was granted by the Papua New Guinea Institute of Medical Research Institutional Review Board (IRB No. 0913) and the Papua New Guinea Medical Research Advisory Council (MRAC No. 10.02). The Ethik-Kommission beider Basel has been informed and had approved the study. Written informed consent was obtained from all study participants. 
Figure 1. Map of TB passive case detection study sites in Papua New Guinea, 2010-2012

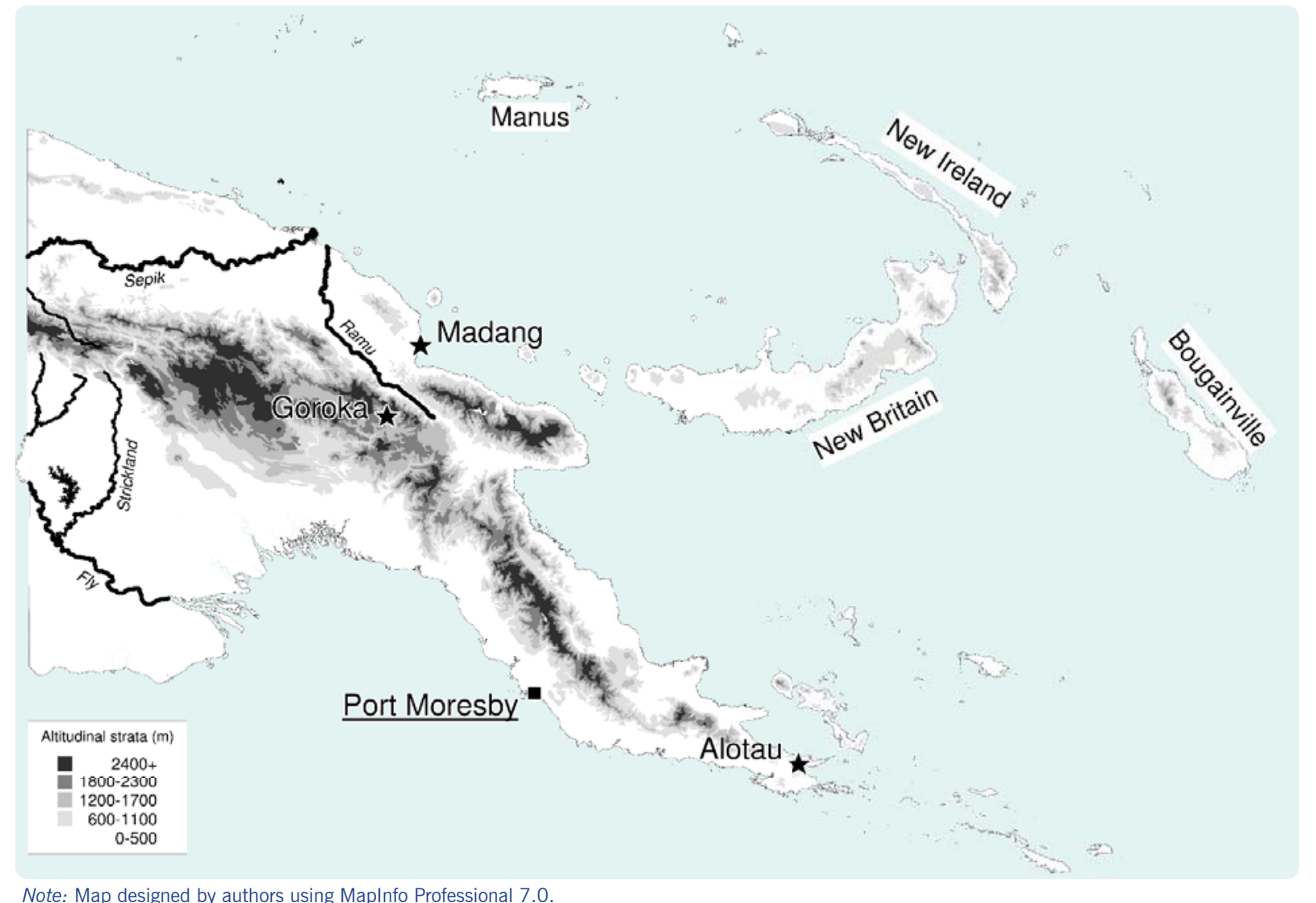

\section{RESULTS}

A total of 396 sputum samples were collected in three provincial hospitals in Papua New Guinea (Figure 1). Of the collected samples, 335 were sent to Australia for culture and 225 samples grew in culture. NTM were detected in $4 \%(9 / 225)$ of those samples. Five $(2.2 \%)$ samples contained a NTM only, consisting of three isolates of $M$. fortuitum, one isolate of $M$. terrae and one isolate of $M$. intracellulare. Four (1.8\%) isolates were identified as mixed cultures containing both bacteria of the MTBC and NTM. These included three cultures of MTBC and M. avium and one culture of MTBC and M. intracellulare (Table 1).

All but one of the NTM infections were detected in females. All the cases with either a mixed infection or a NTM infection only had reported productive coughs for at least two weeks. All the cases with a mixed infection of MTBC and NTM additionally reported weight loss and at least one other symptom, including breathing difficulties $(n=3)$, chest pain $(n=3)$ fever and night sweats $(n=2)$. Among the five cases with an NTM infection only, four cases reported shortness of breath and fever. Three of those cases experienced weight loss and either chest pain or night sweats, or both. The case infected with $M$. intracellulare reported no other symptoms except for productive cough and headache. None of the cases had reported any previous TB episode (Table 1).

\section{DISCUSSION}

To our knowledge this is the first study describing the presence of NTM in Papua New Guinea. In five (2.2\%) of the 225 cases, the isolate was identified as a NTM. Without culture results from at least one more follow-up sample, this may indicate several false-positive TB cases. General symptoms caused by NTM infections cannot be distinguished from symptoms observed in TB cases, and the appearances of the bacteria cannot be differentiated when examined by AFB ZN light microscopy.

It is interesting that in our case cohort all but one NTM isolates were found in females; the only isolate identified in a male was $M$. terrae. There are some NTM species which were more commonly isolated 
Table 1. Characteristics and symptoms reported of the cases with NTM detected in their sputum samples, Papua New Guinea, 2010-2012 $(n=9)$

\begin{tabular}{|c|c|c|c|c|c|c|c|c|}
\hline \multirow{2}{*}{ Sex } & \multirow{2}{*}{$\begin{array}{c}\text { Age } \\
\text { (years) }\end{array}$} & \multirow{2}{*}{$\begin{array}{l}\text { Mycobacterial } \\
\text { strain }\end{array}$} & \multicolumn{5}{|c|}{ Symptoms } & \multirow{2}{*}{$\begin{array}{l}\text { Disease } \\
\text { outcome }\end{array}$} \\
\hline & & & Fever & $\begin{array}{l}\text { Weight } \\
\text { loss }\end{array}$ & $\begin{array}{l}\text { Night } \\
\text { sweat }\end{array}$ & $\begin{array}{l}\text { Breathing } \\
\text { difficulties }\end{array}$ & $\begin{array}{l}\text { Chest } \\
\text { pain }\end{array}$ & \\
\hline Female & 50 & MTBC + M. avium & Yes & Yes & Yes & Yes & No & Defaulted \\
\hline Female & 28 & MTBC + M. avium & No & Yes & No & Yes & No & Lost to follow-up \\
\hline Female & 19 & MTBC + M. avium & Yes & Yes & Yes & No & No & Lost to follow-up \\
\hline Female & 32 & $\begin{array}{c}\text { MTBC }+ \\
\text { M. intracellulare }\end{array}$ & Yes & Yes & No & Yes & Yes & $\begin{array}{l}\text { Treatment } \\
\text { completed }\end{array}$ \\
\hline Female & 18 & M. fortuitum & Yes & Yes & Yes & Yes & Yes & Cured \\
\hline Female & 28 & M. fortuitum & Yes & No & Yes & Yes & No & $\begin{array}{l}\text { Treatment } \\
\text { completed }\end{array}$ \\
\hline Female & 50 & M. fortuitum & Yes & Yes & No & Yes & Yes & Unknown \\
\hline Female & 60 & M. intracellulare & No & No & No & No & No & Cured \\
\hline Male & 36 & M. terrae & Yes & Yes & Yes & No & Yes & Unknown \\
\hline
\end{tabular}

MTBC, Mycobacterium tuberculosis complex; and NTM, non-tuberculous mycobacteria.

from females. ${ }^{2,7,26}$ Another study showed an increased prevalence of funnel chest (pectus excavatum) and abnormal narrowing of the thoracic dimension in female cases infected with NTM of the $M$. avium complex not seen in males. ${ }^{26}$ Also, the so-called Lady Windermere syndrome, a specific pulmonary disorder caused by bacteria of the $M$. avium complex, was only found in women. ${ }^{27}$

There are only a few reports on NTM from TB-endemic countries, ${ }^{3}$ and it is generally difficult to compare our findings with studies from other countries. In a recently published study from Nigeria, for example, $15 \%$ of culture-grown mycobacteria isolated from presumptively diagnosed pulmonary TB cases were NTM. ${ }^{28}$ Compared to that study, a ratio of $2.2 \%$ in our study is relatively low. However, culture criteria of these two studies differed. Whereas in our study only smearpositive samples were cultured. A 2013 study also included smear-negative samples, which turned out to be more strongly associated with NTM infections than smear-positive samples. ${ }^{28}$ It is likely that limiting culture to smear-positive isolates in our study has reduced the chances of detecting NTM in sputum. However, culturing smear-positive samples only is in accordance with the protocols of the National TB Programme of Papua New Guinea and a result of logistic challenges arising from the lack of an in-country culture facility.

Our study population was furthermore limited to suspected pulmonary TB cases aged 15 years or above from three sites within Papua New Guinea, and it is unclear whether inferences can be made to the rest of Papua New Guinea. Nevertheless, compared to the few studies conducted in Papua New Guinea in the 1960s and 1980 s, ${ }^{19-21}$ where tuberculin skin testing did not provide evidence for NTM, our results highlight the existence of NTM in the community and the potential impact on TB diagnosis in the country. While the possibility remains that the presence of NTM in sputum specimens is due to colonization with these environmental organisms, they can also lead to falsepositive TB diagnosis when AFB smear microscopy is used alone. The standard anti-TB treatment is not ideal for NTM, as different antibiotics than the ones used against TB are required to treat $\mathrm{NTM},{ }^{2,10}$ leading to an additional burden for the case as well as the National TB Programme. With an increasing burden of HIV/AIDS, NTM may also become an increasing source of disease, requiring different approaches for case management and treatment.

In Papua New Guinea, the diagnosis of multidrugresistant (MDR) TB was for a long time based on the observation of repeated treatment failure despite compliance with treatment. ${ }^{29}$ Since 2012, TB drug resistance surveillance based on Xpert ${ }^{\circledR}$ MTB/RIF assay (Cepheid, Sunnyvale, California, USA) has started in a few major cities. ${ }^{30}$ However, it probably remains difficult for many health facilities to obtain a culture/DST-confirmed diagnosis of MDR-TB. If the actual cause of treatment failure is not drug resistance, but an NTM infection, 
this would have a major impact on individual case management, especially if the symptoms of the disease are similar to those of MDR-TB. This has been shown in a study from India, where $17.6 \%$ of the suspected MDR pulmonary TB cases were actually NTM infections. ${ }^{3}$ An additional challenge to the laboratory is the presence of mixed infections of NTM and MTBC; reliable DST for MTBC may be difficult if the strain cannot be isolated in pure culture, leading to false positivity including incorrect designation of MDR-TB and extensively drugresistant TB.

As our sample size of detected NTM is small, further studies are required to obtain significant data to establish a valid diagnostic algorithm and treatment guidelines for pulmonary diseases caused by NTM. However, no NTM identification is yet performed in the framework of the National TB Programme in Papua New Guinea, and to date, no biosafety level 3

laboratory required for culturing mycobacteria is available in the country. Samples from cases suspected of having MDR-TB are shipped to a mycobacterium reference laboratory in Australia for culture. In-country mycobacterial culture would distinguish TB from NTM infections much more rapidly and at the same time improve the detection of drug-resistant TB.

It is recommended that NTM infection surveillance could be added to the TB drug resistance surveillance of the National TB Programme. ${ }^{30}$ Data from NTM surveillance would determine NTM's role in pulmonary disease in Papua New Guinea and would inform health authorities to target interventions and response in the future. This would relieve both cases and the health system. As Xpert ${ }^{\circledR}$ MTB/RIF assay is not detecting NTM, smear-positive but Xpert ${ }^{\circledR}$ MTB/RIF-negative results could be used as an indicator for NTM infection and as a basis for further investigation. Until culture becomes available within the country, PCR-based assays amplifying the internal transcribed spacer region of 16-23S rRNA could be implemented at the country's Central Public Health Laboratory to distinguish NTM from MTBC directly from clinical samples. ${ }^{31}$

\section{Conflicts of interest}

None declared.
Funding

This research was conducted in the framework of a TB passive case detection study funded by the Stanley Thomas Johnson Foundation and the Medicor Foundation Liechtenstein.

\section{Acknowledgements}

We thank all study participants who agreed to having their samples collected and analysed. We further thank the health authorities of all three study provinces for approval and general support. We are indebted to the Papua New Guinea Institute of Medical Research study teams and the hospital staff in all three sites for sample collection, diagnosis, access to infrastructure and general support. Also, the contribution of the staff of the Queensland Mycobacterium Reference Laboratory is gratefully acknowledged.

\section{References:}

1. Tortoli E. Impact of genotypic studies on mycobacterial taxonomy: the new mycobacteria of the 1990s. Clinical Microbiology Reviews, 2003, 16:319-354. doi:10.1128/CMR.16.2.319354.2003 pmid: 12692101

2. Griffith DE et al.; ATS Mycobacterial Diseases Subcommittee; American Thoracic Society; Infectious Disease Society of America. An official ATS/IDSA statement: diagnosis, treatment, and prevention of nontuberculous mycobacterial diseases. American Journal of Respiratory and Critical Care Medicine, 2007, 175:367-416. doi:10.1164/rccm.200604-571ST pmid: 17277290

3. Gopinath K, Singh S. Non-tuberculous mycobacteria in TB-endemic countries: are we neglecting the danger? PLoS Neglected Tropical Diseases, 2010, 4:e615. doi:10.1371/journal.pntd.0000615 pmid:20436962

4. Kendall BA et al. Isolation of non-tuberculous mycobacteria from the sputum of patients with active tuberculosis. International Journal Tuberculosis Lung Disease: Official Journal International Union against Tuberculosis and Lung Disease, 2010, 14:654656. pmid:20392362

5. Henry MT et al. Nontuberculous mycobacteria in non-HIV patients: epidemiology, treatment and response. European Respiratory Journal, 2004, 23:741-746. doi:10.1183/09031936.04.0011 4004 pmid: 15176690

6. Huang $\mathrm{JH}$ et al. Mycobacterium avium-intracellulare pulmonary infection in HIV-negative patients without preexisting lung disease: diagnostic and management limitations. Chest, 1999, 115:10331040. doi:10.1378/chest.115.4.1033 pmid:10208205

7. Prince DS et al. Infection with Mycobacterium avium complex in patients without predisposing conditions. New England Journal of Medicine, 1989, 321:863-868. doi:10.1056/ NEJM198909283211304 pmid:2770822 
8. Thomson RM; NTM working group at Queensland TB Control Centre and Queensland Mycobacterial Reference Laboratory. Changing epidemiology of pulmonary nontuberculous mycobacteria infections. Emerging Infectious Diseases, 2010, 16:1576-1583. doi:10.3201/eid1610.091201 pmid:20875283

9. Mdluli $K$ et al. Mechanisms involved in the intrinsic isoniazid resistance of Mycobacterium avium. Molecular Microbiology, 1998, 27:1223-1233. doi:10.1046/j.13652958.1998.00774.x pmid:9570407

10. Root RK, editor. Clinical infectious diseases: a practical approach New York, Oxford University Press, 1999, p. 1013.

11. Diagnostic standards and classification of tuberculosis in adults and children. This official statement of the American Thoracic Society and the Centers for Disease Control and Prevention was adopted by the ATS Board of Directors, July 1999. This statement was endorsed by the Council of the Infectious Disease Society of America, September 1999. American Journal of Respiratory and Critical Care Medicine, 2000, 161:1376-1395. pmid:10764337

12. Bensi EPA, Panunto PC, Ramos M de C. Incidence of tuberculous and non-tuberculous mycobacteria, differentiated by multiplex PCR, in clinical specimens of a large general hospital. Clinics (Sao Paulo, Brazil), 2013, 68:179-184. doi:10.6061/ clinics/2013(02)OA10 pmid:23525313

13. Hwang SM et al. Simultaneous detection of Mycobacterium tuberculosis complex and nontuberculous mycobacteria in respiratory specimens. Tuberculosis (Edinburgh, Scotland), 2013, 93:642-646. doi:10.1016/j.tube.2013.07.007 pmid:23988279

14. Luetkemeyer AF et al.; Adult AIDS Clinical Trials Group A5255 Study Team. Evaluation of two line probe assays for rapid detection of Mycobacterium tuberculosis, tuberculosis (TB) drug resistance, and non-TB mycobacteria in HIV-infected individuals with suspected TB. Journal of Clinical Microbiology, 2014, 52:10521059. doi:10.1128/JCM.02639-13 pmid:24430455

15. van der Werf MJ et al. Inventory study of non-tuberculous mycobacteria in the European Union. BMC Infectious Diseases, 2014, 14:62. doi:10.1186/1471-2334-14-62 pmid:24502462

16. Poyntz $\mathrm{HC}$ et al. Non-tuberculous mycobacteria have diverse effects on BCG efficacy against Mycobacterium tuberculosis. Tuberculosis (Edinburgh, Scotland), 2014, 94:226-237. doi:10.1016/j.tube.2013.12.006 pmid:24572168

17. Fine PE. Variation in protection by BCG: implications of and for heterologous immunity. Lancet, 1995, 346:1339-1345. doi:10.1016/S0140-6736(95)92348-9 pmid:7475776

18. Rieder HL. Methodological issues in the estimation of the tuberculosis problem from tuberculin surveys. International Journal Tuberculosis Lung Disease: Official Journal International Union against Tuberculosis and Lung Disease, 1995, 76:114-121. doi:10.1016/0962-8479(95)90552-9 pmid:7780092

19. Bagshawe $A$ et al. BCG vaccination in leprosy: final results of the trial in Karimui, Papua New Guinea, 1963-79. Bulletin of the World Health Organization, 1989, 67:389-399. pmid:2680140
20. Scott GC, Wigley SG, Russell DA. The Karimui trial of BCG. 2. Tuberculin reactions in a leprosy-endemic but tuberculosisfree population. International journal of leprosy and other mycobacterial diseases: official organ of the International Leprosy Association, 1966, 34:139-146. pmid:5330190

21. Brown P, Cathala F, Gajdusek DC. Mycobacterial and fungal skin sensitivity patterns among remote population groups in Papua New Guinea, and in the New Hebrides, Solomon, and Caroline Islands. The American Journal of Tropical Medicine and Hygiene, 1981, 30:1085-1093. pmid:6792936

22. Ley SD et al. Diversity of Mycobacterium tuberculosis and drug resistance in different provinces of Papua New Guinea. BMC Microbiology, 2014, 14:307. doi:10.1186/s12866-014-0307-2 pmid:25476850

23. Petroff SA. A new and rapid method for the isolation and cultivation of Tubercule bacilli directly from the sputum and feces. Journal of Experimental Medicine, 1915, 21:38-42. doi:10.1084/ jem.21.1.38 pmid:19867850

24. Canetti $G$ et al. Advances in techniques of testing mycobacterial drug sensitivity, and the use of sensitivity tests in tuberculosis control programmes. Bulletin of the World Health Organization, 1969, 41:21-43. pmid:5309084

25. Ballif M et al. Genetic diversity of Mycobacterium tuberculosis in Madang, Papua New Guinea. International journal of leprosy and other mycobacterial diseases: official organ of the International Leprosy Association, 2012, 16:1100-1107. pmid:22710686

26. Iseman MD, Buschman DL, Ackerson LM. Pectus excavatum and scoliosis: thoracic anomalies associated with pulmonary disease caused by Mycobacterium avium complex. American Review of Respiratory Disease, 1991, 144:914-916. doi:10.1164/ ajrccm/144.4.914 pmid:1928970

27. Reich JM, Johnson RE. Mycobacterium avium complex pulmonary disease presenting as an isolated lingular or middle lobe pattern: the Lady Windermere syndrome. Chest, 1992, 101:1605-1609. doi:10.1378/chest.101.6.1605 pmid:1600780

28. Aliyu G et al. Prevalence of non-tuberculous mycobacterial infections among tuberculosis suspects in Nigeria. PLOS ONE, 2013, 8:e63170. doi:10.1371/journal.pone.0063170 pmid:23671669

29. National TB Program Unit, Disease Control Branch, National Department of Health. Papua New Guinea country guidelines for the programmatic management of drug-resistant tuberculosis. Port Moresby, Papua New Guinea, 2011, p. 69.

30. Ley SD, Riley I, Beck H-P. Tuberculosis in Papua New Guinea: from yesterday until today. Microbes and infection/Institut Pasteur, 2014, 16:607-614. doi:10.1016/j.micinf.2014.06.012 pmid:25025486

31. Gopinath K, Singh S. Multiplex PCR assay for simultaneous detection and differentiation of Mycobacterium tuberculosis, Mycobacterium avium complexes and other mycobacterial species directly from clinical specimens. Journal of Applied Microbiology, 2009, 107:425-435. doi:10.1111/j.1365-2672.2009.04218.x pmid:19302308 